\title{
STANDARDS DE TOXIDADE E INSEGURANÇA ALIMENTAR NO BRASIL
}

\author{
Ana Luiza da Gama e Souza
}

RESUMO: A proposta deste artigo é fazer uma análise da problemática da insegurança alimentar no Brasil no que toca a contaminação dos alimentos por substâncias químicas como herbicidas, pesticidas e outros contaminantes. O problema será enfrentado, por um lado, a partir dos standards privados que guiam o sistema alimentar no mundo e por outro, dos indicadores de direitos humanos, cuja finalidade é proporcionar um monitoramento efetivo das obrigações do Estado de garantir a segurança alimentar. Neste artigo propõe-se analisar as implicações dos standards privados de toxidade para a efetividade do monitoramento da obrigação do Estado. A metodologia adotada é bibliográfico-documental.

Palavras-chave: Standards, indicadores, direitos humanos, segurança alimentar

\section{STANDARDS OF TOXICITY AND FOOD INSECURITY IN BRAZIL}

\begin{abstract}
This article aims to analyze the problem of food insecurity in Brazil, regarding contamination of food by chemical substances such as herbicides, pesticides and other contaminants. The problem will be faced, on the one hand, from the private standards guiding the food system in the world and, on the other hand, the human rights indicators, whose purpose is to provide effective monitoring of the State's obligations to ensure food security. In this article we propose to analyze the relation and implication of the private standards of toxicity for the effectiveness of the human rights indicators and so to the state monitoring mechanism. The methodology adopted is bibliographic-documentary.
\end{abstract}

Key-words: Standards, indicators, human rights, food security

\section{INTRODUÇÃO}

O sucesso do monitoramento da segurança alimentar no Brasil, depende de indicadores eficientes que sejam adequados a avaliar no maior grau possível e de forma abrangente o cumprimento da obrigação do Estado, tal como imposta pelas normas internacionais e internas.

Um mecanismo de monitoramento eficiente necessita, então, de indicadores apropriados, desenvolvidos a partir de metodologias eficientes que garantam uma avaliação compreensiva da atuação do Estado, no sentido de garantir não só o acesso em nível

\footnotetext{
${ }^{1}$ Professora titular e pesquisadora do Departamento de Direito da UNESA. Contato: anagama64@gmail.com
} 
quantitativo, mas principalmente qualitativo, garantindo uma alimentação livre de contaminantes que possam comprometer a saúde dos brasileiros. No entanto, os indicadores, para além de uma metodologia hábil, dependem também de standards $^{2}$ voltados para o sistema agroalimentar que apontem e informem precisamente o nível de aceitabilidade ou não aceitabilidade da utilização de produtos químicos na produção de alimentos.

Um indicador adequado e eficiente deve apontar não só a utilização de pesticidas nos processos agroalimentares, mas deve acima de tudo discriminar detalhadamente o tipo, a composição e os níveis de ativos químicos introduzidos no produto, informação relevante a ser cuidadosamente considerada na elaboração de um standard, que a seu turno deve considerar valores como a saúde humana ou ambiental. Esta é a problemática a ser enfrentada neste artigo: como obter avaliações precisas da atuação do Brasil no cumprimento das exigências de segurança alimentar, diante das incertezas acerca do grau de confiabilidade nas informações técnicas disponibilizadas pelos standards?

A insegurança alimentar no Brasil é questão de saúde pública, o que exige um olhar mais atento às questões relacionadas ao monitoramento de direitos humanos, em especial a questão dos indicadores e sua relação com os standards privados de níveis de toxidade.

Neste intento, concluindo-se que indicadores específicos para os agrotóxicos, quando existentes, são totalmente dependentes de standards privados internos e internacionais que estabelecem os níveis de toxidade de acordo com os interesses privados do mercado e que são desenvolvidos cientificamente, utilizando-se de técnicas complexas que muitas vezes comprometem a transparência necessária das informações que contém e que neste sentido estão voltados tanto para evitar riscos à saúde humana, como também para não criar embaraços ao livre comércio das indústrias químicas. Deste modo, sua função como parâmetro para o monitoramento de direitos humanos fica parcialmente prejudicada.

Embora os mercados alternativos estejam ganhando espaço no Brasil, em especial o mercado de produtos frescos e orgânicos, ainda estamos longe de garantir o acesso a todos os brasileiros a uma alimentação não só adequada em termos de quantidade, o que já foi em parte realizado pelos programas de governo ${ }^{3}$, mas em termos de qualidade, o que implica em alimentos livres de qualquer substância, que em qualquer grau possam comprometer a saúde e a vida dos brasileiros. A realização da segurança alimentar depende de mecanismos

\footnotetext{
${ }^{2}$ Standards são medidas pelas quais produtos, processos e produtores são julgados. São eles que definem o que será comercializado, estabelece convenções para ordenar o processo produtivo e fixa níveis de (BUSCH and BING, 2006, p. 3)
} 
inovadores, adequados, eficientes e independentes, de monitoramento dos esforços do Brasil na garantia de uma alimentação saudável.

\section{MÉTODO}

Neste artigo, num primeiro momento foram analisados os relatórios da Comissão de Direitos Humanos da Organização dos Estados Americanos para identificar e analisar o conjunto de indicadores de progresso desenvolvidos pela Comissão, e a nova metodologia utilizada, avaliando a sua eficiência no monitoramento da segurança alimentar no Brasil, atentando para de utilização de pesticidas na produção de alimentos em níveis de toxidade não admitidos pelos órgãos de fiscalização. Paralelamente, analisou-se o mecanismo de monitoramento da segurança alimentar no Brasil, que foi instituído inicialmente pelo Plano Nacional de Segurança Alimentar (PLANSAN) para 2012-2015 e aprimorado pelo II Plano Nacional de Segurança Alimentar para 2016-2019, ambos elaborados pela Câmara Interministerial de Segurança Alimentar e Nutricional (CAISAN), comparando as propostas de monitoramento com os resultados apresentados pelo CAISAN no Relatório Indicadores e Resultados do Plano Nacional de Segurança Alimentar e Nutricional 2012-2015, para avaliar o progresso do Brasil na garantia da segurança alimentar. Num segundo momento, verificouse a necessidade de conhecer e analisar o processo de standarização do sistema agroalimentar, em especial os standards de nível de toxidade, intrinsecamente relacionados ao monitoramento da segurança alimentar, como garantia de uma alimentação livre de pesticidas que sejam prejudiciais à saúde humana.

\section{A INSEGURANÇA ALIMENTAR NO BRASIL}

O Brasil é um dos líderes mundiais no consumo de agrotóxicos, contabilizando inúmeros casos, inclusive de contaminação aguda ${ }^{4}$ por pesticidas classificados pela Organização Mundial de Saúde (OMS) como sendo de mais alto risco e periculosidade para a saúde humana. ${ }^{5}$ Embora a utilização de pesticidas não seja uma prática recomendável ${ }^{6}$, no Brasil ela ainda é realizada em larga escala, não se limitando a áreas rurais, como reconhecido

\footnotetext{
${ }^{4}$ Cf. em http://www.who.int/bulletin/volumes/86/3/07-041814/en/

${ }^{5}$ FARIA, N.; FASSE, A. C. G. e FACHINI. 2007.

${ }^{6}$ Tendo em vista os efeitos danosos dos agrotóxicos, a agricultura ecológica (orgânica) vem sendo recomendada como alternativa. Cf. em http://www.fao.org/organicag/oa-home/fr/
} 
pela ANVISA na Nota sobre o Uso de Agrotóxicos em área urbana ${ }^{7}$ e nem aos produtos autorizados pelos órgãos competentes de fiscalização e controle.

No último relatório da ANVISA, 36\% das amostras apresentaram as seguintes irregularidades: a) presença de agrotóxicos em níveis acima do LMR em 38 amostras, correspondendo a 2,3\% do total; b) constatação de agrotóxicos não autorizados (NA) para a cultura em 520 amostras, correspondendo a 32\% do total; c) resíduos acima do LMR e NA simultaneamente em 31 amostras, correspondendo a 1,9\% do total. ${ }^{8}$ A Portaria SDA $\mathrm{n}^{\mathrm{o}} 115$, de 30 de agosto de 2013 do Ministério da Agricultura, Pecuária e Abastecimento (MAPA) ${ }^{9}$ aponta também a presença de pesticidas em alimentos que diariamente chegam à mesa dos brasileiros, dentre eles o Carbofurano ${ }^{10}$ e Glifosato ${ }^{11}$, classificado pela organização mundial de saúde como sendo altamente perigoso.

\section{MONITORAMENTO DA SEGURANÇA ALIMENTAR: A QUESTÃo DOS INDICADORES DE DIREITOS HUMANOS}

Os direitos humanos estabelecem padrões morais para guiar a ação dos Estados e mais recentemente em 2011, também as corporações transnacionais ${ }^{12}$. De certo modo são standards, mas estritamente ligados a valores, moralmente considerados. Direcionados aos Estados, a garantia de uma alimentação livre de pesticidas está prevista em diversos acordos como o Pacto Internacional de Direitos Econômicos, Sociais e Culturais e o Pacto Interamericano de Direitos Econômicos Sociais e Culturais, este último inclusive estabelece a obrigação do Estado de realizar progressivamente a segurança alimentar, o que possibilitou a criação de mecanismos de monitoramento do cumprimento desta obrigação No entanto, avaliar o avanço em segurança alimentar conecta o Estado aos direitos humanos, ao sistema agroalimentar e seus standards. Nesse sentido, conjuga as demandas de direitos dirigidas

\footnotetext{
${ }^{7}$ http://ultimosegundo.ig.com.br/brasil/2014-01-10/pesquisadores-alertam-para-expansao-de-transgenicos-eagrotoxicos-no-brasil.html.

${ }^{8} \mathrm{http} / /$ portal.anvisa.gov.br/wps/wcm/connect/d480f50041ebb7a09db8bd3e2b7e7e4d/Relat\%C3\%B3rio\%2BPA RA\%2B2011-12\%2B-\%2B30_10_13 1.pdf?MOD=AJPERES. p. 18.

${ }^{9} \mathrm{ftp}: / / \mathrm{ftp}$. saude.sp.gov.br/ftpsessp/bibliote/informe_eletronico/2013/iels.set.13/Iels165/U_PT-MAPA-SDA115_300813.pdf

${ }^{10}$ Cf. WORLD HEALTH ORGANIZATION (WHO). 2009. The Recommended Classification of Pesticides by Hazard and Guideline for Classification. WHO Library Cataloguing-in-Publication-data, p. 21.

${ }^{11}$ United Nations. Organization for Food and Agriculture. FAO specifications and evaluations for agricultural pesticides. GLYPHOSATE (N-(phosphonomethyl)glycine). 2016.

${ }^{12}$ UNITED NATIONS, 2011
} 
prioritariamente aos Estados, com as regulações e standarização privadas, oriundas das instituições do mercado.

O Pacto Internacional de Direitos Econômicos, Sociais e Culturais e o Protocolo de São Salvador estabelecem o compromisso dos Estado em realizar progressivamente os direitos econômicos e sociais enumerados em seus textos, dentre eles o direito à alimentação adequada. No Protocolo de São Salvador os Estados garantem expressamente o direito à nutrição adequada e comprometem-se em aperfeiçoar os métodos de produção, abastecimento e distribuição de alimentos, o que significa que esta norma internacional vincula diretamente os Estados e indiretamente o sistema agroalimentar ${ }^{13}$.São bem claros os limites da obrigação de resultado do Estado: realizar o direito à nutrição adequada, garantindo o "mais alto nível de bem-estar físico"14 e para tal compromete-se em aperfeiçoar os métodos de produção dos alimentos, proibindo, fiscalizando e controlando a produção dos alimentos que devem estar de substâncias nocivas à saúde humana.

O monitoramento do direito à alimentação adequada no Brasil, diante do grave problema de saúde pública, compreende sobretudo, para além das normas, um conjunto de políticas públicas eficientes no combate a utilização de pesticidas na produção agrícola e industrial brasileira que depende da definição de standards que delimitem o espaço dos pesticidas no mercado e o próprio mercado de pesticidas. O próprio contexto brasileiro de corrupção na máquina administrativa e das políticas claramente favoráveis aos investimentos do mercado das indústrias químicas são ingredientes que dificultam o monitoramento.

O sucesso do mecanismo de monitoramento da s obrigações do Estado com meio de avaliar e assim garantir a realização dos direitos humanos no Brasil, depende da escolha de indicadores, ferramentas analíticas que são a espinha dorsal do acompanhamento do grau de realização dos direitos humanos econômicos e sociais em nível local, nacional, regional ou global. No entanto, o desenvolvimento de indicadores eficientes e adequados às diversas realidades e contextos é também uma difícil tarefa. No que se refere à segurança alimentar é ainda maior o desafio, como reconhece a ONU, pois o desenvolvimento de um indicador de poluição química é tarefa complexa diante da dificuldade de medição em termos internacionalmente comparáveis. ${ }^{15}$

\footnotetext{
${ }^{13}$ Art. 12 do Protocolo de San Salvador.

${ }^{14}$ Art. 10 do Protocolo de San Salvador.

${ }^{15}$ UN. 2012. p. 162.
} 
Grenn define indicador como "uma peça de informação usada na medição da extensão a qual um direito está sendo realizado ou gozada em uma dada situação"16 Tecnicamente o termo significa um conjunto de estatísticas que podem servir como uma metáfora para um fenômeno que não pode ser mensurado e difere-se, na literatura dos direitos humanos, do termo benchmarks que são objetivos ou metas específicas para circunstâncias peculiares de cada Estado, ou seja, são limites mínimos de condições que não podem ser ultrapassadas nos contextos sócio econômicos avaliados (GREEN. 2001, p. 1076 e 1080).

Tradicionalmente, nos processos de monitoramento tem se utilizado indicadores de progresso $^{17}$ que são ferramentas úteis para medir performances diante de determinado padrão (benchmarks). São utilizados para avaliar e planificar o progresso de determinada situação ao longo do tempo, fixar algumas metas e medir a evolução destas metas, tendo como base estatísticas (dados duros) que permitem refletir sobre o progresso ou não de determinada situação. Indicadores de progresso apontam melhoras materiais, o que não implica em mostrar a melhora na qualidade da vida das pessoas, o que depende de outros vários fatores, tendo em vista a integralidade dos direitos humanos.

$\mathrm{Na}$ aplicação de indicadores de direitos são utilizados dados da situação social e econômica do país como referência para a análise das obrigações progressivas deste país. Este dado pode ser complementado por outros dados referentes aos mecanismos institucionais e políticas públicas, como também aos recursos e capacidade de que dispõe a população para exigir os direitos.

Susan Randolph and Shareen Hertel (2012, p. 6) classificam os indicadores em três instâncias: a) indicadores estruturais, que refletem os compromissos em nível global tratados, declarações, agendas - e em nível nacional - tratados ratificados, normas, estrutura institucional e programas de ação necessários à realização do direito; b) indicadores de processo, que refletem o esforço do Estado na realização do direito, levando-se em conta os diversos fatores que afetam realização do direito; c) Indicadores de resultado, focados diretamente na extensão na qual o direito é realizado. No entanto, indicadores com este perfil tem um problema prático, que é o foco nos direitos (right bearer). Tanto os indicadores estruturais, como os de processo e de resultado normalmente avaliam o grau de fruição e gozo de direitos dentro dos limites do Estado (right-bearer), quando deveriam avaliar em que

\footnotetext{
${ }^{16}$ GREEN. 2001, P. 1065

${ }^{17}$ ORGANIZATION OF AMERICAN STATES .2015.
} 
medida os Estados estão cumprindo suas obrigações mais amplas em termos de direitos humanos (duty-bearer).

\section{OS INDICADORES DE PROGRESSO PREVISTOS NO PROTOCOLO DE SÃO SALVADOR: A NOVA METODOLOGIA.}

Comprometendo-se a dar eficácia ao artigo 19 do PSS, em 2005, a Assembleia Geral da OEA aprovou através da Resolução 2074-XXXV-O / 05 o guia para a preparação dos relatórios/informes periódicos previstos no art. 19 do Protocolo de São Salvador ${ }^{18}$, orientando o Conselho Permanente a compor e dar início ao Grupo de Trabalho incumbido de definir e selecionar os indicadores que serão utilizados pelos Estados na elaboração de seus informes.

Na mesma Resolução, a Assembleia Geral, seguindo o PSS, estabelece que os relatórios a serem produzidos deverão ser guiados pelo princípio da progressividade e por um sistema de indicadores de progresso específicos para cada grupo de direitos econômicos e sociais, sobre os quais as informações serão fornecidas.

Reconhecendo as dificuldades da elaboração de indicadores de direitos humanos específicos para os direitos econômicos e sociais, em 2008, a Comissão Interamericana de Direitos Humanos apresenta um Guia para a preparação de indicadores de progresso, no qual apresenta os problemas a serem enfrentados e propõe uma nova metodologia para monitoramento dos direitos previsto no Protocolo de São Salvador (PSS).

São dois os principais problemas a serem enfrentados pelo Grupo de Trabalho. O primeiro problema consiste no fato de que o PSS não é claro quanto aos critérios que devem nortear o modelo de indicadores de progresso, mas apenas assinala que eles devem permitir determinar a distância entre a situação da realidade e o padrão ou meta desejada pelo Protocolo. O segundo, se refere a dificuldade na definição de uma metodologia adequada que dê conta das especificidades das obrigações contidas no Protocolo, em especial sua natureza inter-relacional.

$\mathrm{Na}$ construção de uma metodologia que seja adequada à proposta de monitoramento do PPS, a Comissão opta por indicadores de direitos e não pelos tradicionais modelos de indicadores de desenvolvimento econômico e social, já que o objetivo dos informes é o de avaliar o grau de cumprimento de obrigações previstas em um tratado de direitos humanos e assim não podem se restringir a dados sobre a situação econômica e social dos Estados, mas 
devem ir além para apurar o grau de cumprimento e efetividade destes direitos econômicos, sociais e culturais.

No entanto, a Comissão não descarta os dados sobre a situação econômica e social, reconhecendo sua importância na avaliação global, quando analisados em conjunto com outros dados, relativos aos mecanismos institucionais e políticas públicas e complementados ainda com dados sobre recursos e capacidades com os quais o Estado conta para garantir uma progressiva efetividade dos direitos garantidos no PSS.

Informações sobre fatores estruturais são também considerados na construção dos indicadores. Estes fatores garantem a possibilidade de acesso efetivo aos direitos, dentre eles estão os fatores normativos, constitucionais e infraconstitucionais, os mecanismos de transparências nas contas públicas, o perfil das políticas de Estado e os programas de ação, bem como os serviços disponíveis pelo Estado. A Comissão reconhece também a importância da avaliação sobre a capacidade das pessoas ${ }^{19}$ de exigir os direitos contemplados no PSS dos quais são titulares.

A Comissão define três tipos de indicadores qualitativos: os estruturais, os relacionados a processos e os relacionado a resultados. Os indicadores estruturais coletam informações de forma a avaliar como o aparato do Estado e o sistema normativo são organizados para realizar os direitos previstos no PSS. Significa, avaliar quais padrões normativos, estratégias, planos e programas de ação e ainda quais órgãos ou agencias foram criados para implementar estes direitos.

Avaliadas as condições estruturais, os indicadores de progresso têm por finalidade monitorar diretamente as políticas públicas do Estado indicando a qualidade e extensão dos esforços em implementar os direitos garantidos pelo PSS, através de medições do âmbito, cobertura e conteúdo das estratégias, planos, programas de ação ou outras atividades do Estados consideradas relevantes para realização dos referidos direitos.

Por último, os indicadores de resultado, cuja função é a medir como as políticas públicas empreendidas pelo Estado impactam nos aspectos que determinam o quão efetiva é um direito previsto no PSS. Estes indicadores medem a performance do Estado no cumprimento do seu dever de garantir a segurança alimentar em termos de realização progressiva. A melhoria nos indicadores de resultado, indicam uma maior efetividade.

\footnotetext{
18 OEA. 2008.

${ }^{19} \mathrm{O}$ entendimento é o que de a obrigação do Estado é garantir os direitos econômicos, sociais e culturais a todas as pessoas e não a muitos ou à maioria.
} 
Para melhorar a análise e organização das informações coletadas, a Comissão sugere a divisão destes indicadores em três categorias: 1) recepção do direito pelo sistema normativo do Estado, pelo aparato institucional e pelas políticas públicas, na teoria e na prática; 2) A capacidade do Estado; 3) O contexto financeiro básico. Todos os três são avaliados sob o prisma estrutural, de processo e de resultado.

A primeira categoria tem por objetivo verificar o nível das disposições - normas constitucionais, infraconstitucionais, jurisprudência, programas de governo e práticas de governo - que reconhecem o direito, o grau de precisão com que é definido e sua efetividade. Também são considerados o nível de exercício do direito pelos seus titulares e a forma como são efetivados, por exemplo, se exigidos diretamente do governo ou por intermédio do judiciário, o que exige verificar também as garantias e os procedimentos disponíveis no caso de violação das obrigações do Estado. Em suma, é preciso que o direito faça parte da lógica e a própria razão das políticas do Estado.

No caso do direito à alimentação adequada, em nível federal, o indicador estrutural de recepção do direito apontaria: 1) se o direito é previsto na Constituição Federal; 2) se é efetivo ou não, significa dizer se está apto a gerar efeitos. Já o indicador de processo de recepção do direito à alimentação adequada avaliaria a existência ou não de jurisprudência relevante sobre o tema e o alcance e abrangência das políticas públicas implementadas para a realização deste direito, incluindo não só as políticas para garantir o acesso como também a qualidade da alimentação, o que significa levar em conta os standards voltados para o sistema agroalimentar, como o LMR (Limite Máximo de Resíduos em pesticidas).

A segunda categoria se refere a capacidade do Estado em termo de instrumental técnico e de distribuição dos recursos do governo dentro do aparato do Estado. O objetivo é avaliar como e de acordo com que parâmetro o governo lida como questão socialmente problemáticas, sobretudo como estabelece seus objetivos e desenvolve suas estratégias e o quanto os parâmetros de implementação dos direitos contidos no PSS são considerados nestes objetivos e estratégias. Isto enseja rever as regras do jogo no aparato do Estado, as relações entre as agências estatais, alocação de tarefas, capacidade financeira a as habilidades dos agentes que irão realizar as tarefas "20, estes, no caso da segurança alimentar, devem estar preparados para enfrentar as exigências técnicas para a compreensão dos standards a serem respeitados. 
A existência de um determinado órgão de fiscalização da realização do direito é um indicador estrutural da capacidade do Estado. No caso da segurança alimentar no Brasil, a existência da Agência Nacional de Vigilância Sanitária (ANVISA) seria um indicador desta natureza. Como também as dotações orçamentárias ${ }^{21}$ para custear as despesas desta agência e a utilização na efetiva implementação das políticas públicas de fiscalização da qualidade dos alimentos produzidos e distribuídos no Brasil, o que também depende de standards adequados a garantir a vida e à saúde humana. Como a medição se estende à qualidade e ao escopo das políticas públicas em um dado período de tempo, a existência de supervisão e avaliação do trabalho das agências de serviços sociais e dos programas de ação e a capacidade do Estado para implementar políticas anticorrupção são também um aspecto da medida da capacidade do Estado. Em suma, segundo a Comissão, a inclusão da categoria "capacidade do Estado" tem a finalidade de obter informações sobre aspectos centrais sobre o quanto as políticas de Estado para a segurança alimentar estão se materializando.

Este modelo de monitoramento proposto pela Comissão foi colocado em prática pelo Grupo de Trabalho em duas etapas ${ }^{22}$, em dois relatórios finalizados respectivamente em $2011^{23}$ e em $2014^{24}$. Em 2011, o Grupo de Trabalho divulgou unicamente os indicadores de progresso para o primeiro grupo - direito à seguridade social, à saúde e à educação - e em 2014 conclui relatório acrescentando o segundo grupo de direitos - direito ao trabalho e sindical, à alimentação adequada, ao meio ambiente saudável e direitos culturais. Ambos os relatórios apresentam indicadores concretos para a avaliação do progresso na realização dos direitos econômicos e sociais contemplados no PSS.

O documento apresentado pelo Grupo de Trabalho se refere à definição de alimentação adequada do art. 12 do $\operatorname{PSS}^{25}$, que, como ponto de partida, deixa algumas incertezas, como o que seja "o mais alto nível físico, emocional e intelectual”, mas, de certo modo, estas imprecisões são enfrentadas pelo grupo de trabalho que reconhece que a prescrição normativa deve ser interpretada em sentido amplo, compreendendo não só o acesso

\footnotetext{
${ }^{21} \mathrm{Em} 2014$, o valor foi de $\mathrm{R} \$ 68.991 .981,85$, conforme o note técnica $1 / 2014$. Cf. http://www.conass.org.br/Notas\%20tecnicas\%202014/NT\%2001-\%202014\%20-\%20VISA.pdf

${ }^{22}$ O Grupo de trabalho optou pela divisão dos direitos contemplados no Protocolo em dois grupos. No primeiro, o direito à saúde, à segurança social e à educação e no segundo, direito ao trabalho, direitos sindicais, direito à alimentação adequada, à saúde ambiental e os benefícios da cultura. Esta divisão reflete a intenção do grupo de trabalho de definir e legitimar indicadores em fases e ao mesmo tempo garantir aos Estados o tempo necessário para que informar os dados necessários para o cálculo dos indicadores

${ }^{23}$ OEA. 2011.

${ }^{24}$ OEA. 2015.

25 "Toda pessoa tem direito a uma nutrição adequada que assegure a possibilidade de gozar do mais alto nível de desenvolvimento físico, emocional e intelectual.”
} 
ao alimento (quantidade), como também a qualidade do alimento, que deve estar livre de substâncias adversas ${ }^{26}$.

Neste sentido faz-se uma primeira consideração: os indicadores devem apontar para fatores estruturais, de processo e de resultado, em todas as três categorias, que indiquem progresso do Estado no sentido do cumprimento de seu dever em direta relação com o sentido amplo do direito a que corresponde. Políticas públicas focadas exclusivamente no combate à fome são fragmentadas, no sentido de que consideram apenas o aspecto quantitativo do direito à alimentação adequada. E a falta de uma política pública mais abrangente afeta ainda outros direitos da mesma natureza, dada a inter-relação do direito à alimentação adequada com o direito à saúde, não só humana, como ambiental.

A segunda consideração mais específica sobre os indicadores é que no item 'recepção

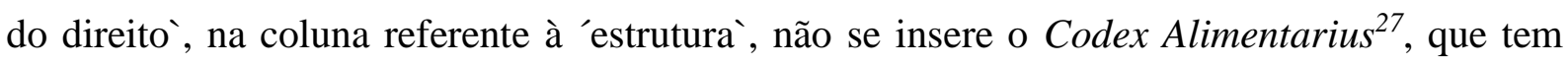
por finalidade harmonizar os standards alimentares internacionais voltados para a proteção da saúde do consumidor e para a promoção práticas de comercio justo ${ }^{28}$ e no mesmo item, na coluna 'processos', não se encontra o indicador "Eliminação, redução ou adequação dos pesticidas aos LRM" ${ }^{29}$, o que não se pode deduzir de nenhum dos outros elementos incluídos nesta categoria. Sua falta implica em graves consequências na avaliação dos resultados na recepção do direito, como no caso do Brasil, já que a Constituição Brasileira ${ }^{30}$ não é específica quanto à obrigação de garantir não só o acesso, como a qualidade dos alimentos. $\mathrm{O}$ direito a uma alimentação livre de pesticidas não é expressamente garantido na legislação brasileira, do que decorre que no processo de recepção do direito deve existir um indicador que aponte para a recepção deste direito e possibilite a avaliação da existência de políticas públicas no sentido de eliminação, redução ou adequação destes contaminantes dos alimentos entregues à população, seja em programas sociais, como o Fome Zero, seja pelo mercado de produtores e distribuidores de alimentos.

\footnotetext{
26 "The availability of food in a quantity and quality sufficient to satisfy the dietary needs of individuals, free from adverse substances, and acceptable within a given culture "(UN. 1999, paragraph 8)

${ }^{27}$ Quanto aos pesticidas, se pode observar que apenas a Convenção de Rotterdam sobre o Procedimento de Consentimento Livre e Esclarecido para Certos Produtos Químicos e Pesticidas Perigosos no Comércio Internacional é considerada como direito recepcionado, mas relacionado ao direito à saúde. No entanto, o próprio direito à alimentação adequada exige o dever de garantir alimentos sem contaminantes.

${ }^{28}$ OEA. 2015, p. 90.

${ }^{29}$ Idem.

${ }^{30}$ Art. $6^{\circ}$ da CRFB.
} 
No entanto, no item 'capacidade do Estado ${ }^{-31}$ pode-se inferir um indicador importante para a garantia de uma alimentação livre de substâncias tóxicas. O primeiro, indicador de 'estrutura', refere-se à existência de um programa sobre qualidade na alimentação e promoção da alimentação saudável em conexão com doenças crônicas não transmissíveis e o segundo, de 'processo', a existência de padrões de uso de pesticidas e agrotóxicos emitidos por autoridade do governo e por empresas privadas e ainda a existência de mecanismo de denúncia e arquivamento. Neste último caso, o indicador limita-se aos padrões emitidos por autoridades do governo, deixando de lado, especificamente, os standards internacionais, que embora não sejam suficientes isoladamente, são necessários para agregar informações.

É fato que a indústrias químicas são parte de um poderoso mercado que avança sobre os interesses do Estados, em especial sobre as políticas de restrição às atividades destas industrias dentro do território brasileiro e talvez na efetividade das políticas de fiscalização na produção e distribuição dos pesticidas. É fato também - o que já foi inclusive comentado acima - que no Brasil ainda são utilizados pesticidas proibidos pela Organização Mundial de Saúde e neste sentido há necessidade de indicadores mais especificamente desenhados para monitorar este tipo de descumprimento da obrigação de realizar o direito à alimentação adequada

\section{MONITORAMENTO E INDICADORES NO BRASIL: O PLANO NACIONAL DE SEGURANÇA ALIMENTAR}

A segurança alimentar no Brasil é de responsabilidade do Ministério da Agricultura, Pecuária e Abastecimento (MAPA), através da Secretaria de Proteção à Agricultura (SPA), órgão que tem autoridade para controlar os aspectos fitossanitários da produção e comércio internacional de toda a pecuária, frutos, vegetais, grãos, plantas, medicamentos veterinários, pesticidas e os componentes. A SPA também registra e inspeciona produtos e atividades que usam organismos geneticamente modificados, em nome da Comissão Técnica Nacional de Biotecnologia (CTNBio), que emite a autorização relevante. No entanto, incumbe a Agência Nacional de Vigilância Sanitária (ANVISA) do Brasil, entidade autônoma relacionada ao Ministério da Saúde controlar a produção e comercialização de produtos e serviços sujeitos a vigilância sanitária para a proteção da saúde humana, sendo responsável pela aprovação e

\footnotetext{
${ }^{31}$ Idem, p. 92.
} 
importação de produtos alimentícios e realizar inspeções sanitárias nos pontos de entrada do Brasil. $^{32}$

O mecanismo de monitoramento da segurança alimentar no Brasil foi instituído inicialmente pelo Plano Nacional de Segurança Alimentar (PLANSAN) para 2012-2015 e aprimorado pelo II Plano Nacional de Segurança Alimentar para 2016-2019, elaborados pela Câmara Interministerial de Segurança Alimentar e Nutricional (CAISAN), que inova na apresentação de novas metodologias de monitoramento voltadas para em especial para a ampliação e fortalecimento de sistemas de produção de alimentos de bases mais sustentáveis, o crescente aumento do sobrepeso/ obesidade e das doenças crônicas não transmissíveis, a promoção da oferta a alimentos saudáveis para toda a população, e a insegurança alimentar e nutricional de populações tradicionais e específicas.

O primeiro PLANSAN, no compromete-se a "aperfeiçoar os mecanismos de gestão, controle e educação voltados para o uso de agrotóxicos, organismos geneticamente modificados e demais insumos agrícolas"33 e para tanto estabelece metas prioritárias para 2012-2015, dentre elas: aumentar em 25\% o número de culturas agrícolas a serem analisadas quanto aos níveis de resíduos de agrotóxicos no âmbito do Programa de Análise de Resíduos de Agrotóxicos; disponibilizar à sociedade notas técnicas de 8 ingredientes ativos de agrotóxicos submetidos à

Reavaliação devido ao elevado perigo que representam para a saúde dos trabalhadores; fortalecer e ampliar o Grupo de Educação e Saúde sobre Agrotóxicos; criar um grupo Intersetorial para a definição de estratégias de controle e uso dos agrotóxicos; ampliar a capacidade de avaliação e registro de agrotóxicos genéricos com a qualificação de mão de obra e sistemas eletrônicos e construir um índice de risco dos insumos agrícolas.

O Plano Nacional de Segurança Alimentar e Nutricional 2012-2105 atribui ao IBAMA a iniciativa de Avaliar a periculosidade e fazer o controle de produtos, substâncias químicas e resíduos perigosos ${ }^{34}$. Este órgão atua em conjunto com a Secretaria de Defesa Agropecuária do Ministério da Agricultura, Pecuária e Abastecimento (MAPA) e a Agência Nacional de Vigilância Sanitária (ANVISA). Em 2012 o IBAMA apresentou o Boletim de Comercialização de Agrotóxicos e Afins - 2000-2012. Neste Boletim, o IBAMA mostra que o número de vendas de produtos químicos muito perigosos e perigosos ao meio ambiente aumentou quase 4\%, de 2009 para 2012, enquanto que a venda de produtos pouco perigosos

\footnotetext{
32 OCDE-FAO. Perspectivas agrícolas 2015-2024.

${ }^{33}$ PLANSAN, 2011
} 
diminui $3,15 \%$. As análises feitas implicam diretamente em standards de toxicidade. No mesmo sentido o indicador proposto pelo PLANSAN, para substâncias químicas ${ }^{36}$ que se refere ao percentual de Contaminação de alimentos por agrotóxicos (amostras irregulares)

Em 2015, o CAISAN apresenta o relatório Indicadores e Resultados do Plano Nacional de Segurança Alimentar e Nutricional 2012-2015 $5^{37}$, utilizando-se de dados quantitativos é claramente centrado no acesso à alimentação e à água, não apresentando o resultado esperado no que se refere à temática dos agrotóxicos. No tópico que se refere a diretriz 3 do I PLANSAN ${ }^{38}$ o relatório mostra o aumento significativo do consumo de frutas e hortaliças como indicativo de uma melhora no hábito alimentar, no entanto não leva em consideração a qualidade das frutas e hortaliças no que concerne a contaminação por agrotóxicos. Ao tratar da diretriz 5, também não são apresentados resultados relativos à utilização de agrotóxicos. É de se notar que as palavras "agrotóxico", "pesticida" ou "substâncias químicas não aparecem uma só vez no texto do relatório.

Em maio de 2016 é divulgado o II PLANSAN para 2016-2019, que inova na intersetorialidade, propondo um monitoramento sistemático dos programas e ações. Reavaliando o PLANSAN 2012-2015, o segundo Plano buscou selecionar dentre o vasto leque de metas do primeiro Plano um conjunto de metas estratégicas e prioritárias, sem, contudo, perder a amplitude do Plano original. O resultado foi a reorganização em 38 objetivos, com 144 metas anualizadas.

A metodologia do II PLANSAN se assenta em 9 Desafios (dimensão mais estratégica do Plano), 121 Metas (resultado final a ser alcançado nos próximos quatro anos, podendo ser de natureza quantitativa ou qualitativa) e 99 Ações Relacionadas (meios necessários para o alcance das metas). Propõe o PLANSAN 2016-2019 um macro desafio, a promoção de sistemas alimentares saudáveis e sustentáveis ${ }^{39}$.

O II PLANSAN apresenta os indicadores de Segurança Alimentar e Nutricional relacionando-os a cada um dos desafios do Plano, indicando as dimensões de análise a eles associadas. Ao desafio $3^{40}$, que trata da promoção da produção de alimentos saudáveis,

\footnotetext{
${ }^{34} \mathrm{http}$ //www.ibama.gov.br/acesso-a-informacao/plano-plurianual-ppa-2012

${ }^{35}$ IBAMA, 2012, p. 37.

${ }^{36}$ CAISAN, 2011, p. 111

${ }^{37}$ CAISAN, 2015

${ }^{38}$ Instituição de processos permanentes de educação alimentar e nutricional, pesquisa e formação nas áreas de segurança alimentar e nutricional e do direito humano à alimentação adequada

${ }^{39}$ Este desafio corresponde à diretriz 2 do PLANSAN 2012-2015.

40 Promover a produção de alimentos saudáveis e sustentáveis, a estruturação da agricultura familiar e o fortalecimento de sistemas de produção de base agroecológica
} 
associa-se um único indicador, a "Comercialização anual de agrotóxicos e afins, por área plantada - Brasil, grandes regiões e UF”. Já ao desafio 5, que trata da promoção e proteção da alimentação adequada e saudável da População Brasileira, associa-se um indicador voltado para a análise dos níveis de resíduos de agrotóxicos nos alimentos de origem vegetal, medição que também depende de standards privados.

Não obstante a inexistência de inovações substanciais no monitoramento da utilização de agrotóxicos trazidas pelo PLANSAN 2016-2019, algumas metas podem ser consideradas relevantes, desde que a avaliação do resultado seja consistente e baseada em indicadores adequados. No tópico "Controle dos riscos relacionados ao consumo de alimentos e a exposição ao uso de agrotóxicos", ressalta-se a meta de reavaliação de 11 ingredientes ativos de produtos agrotóxicos já registrados, considerando novos indícios de risco à saúde humana e a de revisão da a norma que determina os procedimentos para reavaliação toxicológica de ingredientes ativos de agrotóxicos com novos indícios de riscos à saúde humana (RDC Anvisa $n^{\circ}$ 48/2008), embora ainda impliquem na dependência dos standards de nível de toxidade que são a seu turno, uma problemática à parte, como já se mostrou acima.

\section{STRANDARIZAÇÃO DOS NÍVEIS DE TOXIDADE DOS PESTICIDAS}

Standards são medidas pelas quais produtos, processos e produtores são julgados e escalas são categorias usadas para implementar estes standards. Hoje os standards e as escalas formais estão por toda a parte no mundo e poderosos afetam a cadeia de produção, a condição das coisas e o julgamento da atitude das pessoas e de seu mérito e valor. Standards definem o que será comercializado, estabelece convenções para ordenar o processo produtivo e fixa níveis de qualidade (o que não implica necessariamente em alta qualidade). Produtos químicos, como os pesticidas, são categorizados em standards e escalas (BUSCH and BING, 2006, p. 3) em nível local e global, sendo definidos tanto por órgãos de governo, como no caso brasileiro a Agência Nacional de Vigilância Sanitária (ANVISA), quanto por associações de indústrias, como a Associação Brasileira de Indústrias Químicas (ABIQUIM), organizações não-governamentais e organizações internacionais, como a Organização Internacional de Saúde da ONU e o Fórum Econômico Mundial.

Recentemente standards tem sido relacionado à diferenciação de produto. Na indústria de alimentos a diferenciação de produtos é praticada desde a metade do século XX, quando as 
deixaram de oferecer poucos produtos para fornecer um leque imenso de alimentos processados. Segundo Busch and Binden (2006, p. 8), "that diversity was achieved by the creation of myriad standards, each differentiated from others." A mudança mais recente no sistema alimentar é o desenvolvimento e execução de standards privados pelas cadeias globais de supermercados.

A questão que se coloca é que, determina os standards e se eles podem ser concebidos fora de um contexto ético-social mais abrangente, o que poderia significar que os standards são medidas desembebbed, ou seja, concebidas à margem de demandas sociais e morais. Segundo Busch and Bingen (2006), os standards estão associados a questões éticas e de valores, como justiça, direitos, risco e virtudes.

Fligstein (2005, p. 224) adverte sobre a existência em uma dada época de um conjunto de estratégias, táticas e valores compartilhadas que produzem lucro para as maiores corporações e que estas estratégias estão baseadas em um entendimento ou convenções sobre o que é bom para fazer mais dinheiro. Podemos então lançar como hipótese motivadora a de que os standards e valores da indústria de alimentos são formulados nas concepções de controle e refletem o poder das indústrias químicas no mercado de alimentos, o que eleva o risco de violação do direito à alimentação adequada e acarreta a necessidade de mecanismo de monitoramento das obrigações do Estado de garantir a segurança alimentar.

Com relação ao risco decorrente da prática de determinado mercado, como o agroalimentar que analisamos no presente trabalho, a utilização de substâncias tóxicas deve ser informação relevante a ser cuidadosamente considerada na elaboração de um standard, pois diretamente relacionados a valores como a saúde humana ou ambiental. No entanto, o desacordo científico quanto ao nível aceitável de risco na utilização de substâncias tóxicas ou mesmo sobre a proibição do uso ainda é uma realidade e seu enfrentamento é uma questão de interesse público, pois afeta bens públicos indisponíveis. O debate científico sobre o risco e sua avaliação é intenso. Dividido entre os que argumentam que é a probabilidade de dano que define o risco, enquanto outros argumentam que o risco tem várias dimensões e que nenhuma delas é essencial à sua caracterização. ${ }^{41}$

Os standards definem em qual nível o risco é tolerado ou aceitável. No caso da alimentação e diante das exigências de segurança alimentar, este nível pode ser definido em função da consequência que provoca, como doenças, degradação ambiental ou morte ou em função das vulnerabilidades de determinados grupos (idosos, gestantes), mais suscetíveis ou 
ainda segundo outros critérios de avaliação. O que define ou quem define os critérios ou a opção de um e exclusão de outro critério de avaliação de risco é questão aberta, mas por certo esta lacuna implica numa desconexão dos standards com o contexto ético-social mais abrangente.

Recentemente $^{42}$, em Genebra, foi realizado o Workshop conjunto (JMPR) da Organização Mundial do Comercio (OMC) e da Organização para a alimentação e agricultura (OAA) que tem como objetivo geral melhorar o conhecimento e as competências dos funcionários governamentais dos países membros em desenvolvimento e observadores da OMC na implementação do Acordo sobre Medidas Sanitárias e Fitossanitárias (SPS), que trata da forma como os governos podem aplicar as medidas de segurança alimentar e medidas de saúde para animais e plantas. O evento deste ano promoveu-se uma discussão aprofundada, a nível técnico, sobre os teores máximos de resíduos em produtos alimentares (Níveis Máximos de Resíduos de Pesticidas - LMR).

Sob o ponto de vista científico, no painel apresentado pelo Dr. Jürg $\operatorname{Zarn}^{43}$ da Departamento de Segurança Alimentar da Suíça, ele conclui que produtos alimentares seguros se os Limites Máximos de Resíduos puderem ser definidos garantindo que a exposição ao risco seja menor ou igual ao nível de ingestão diária aceitável (ADI) e à dose de referência aguda (ARfD), mesmo quando os padrões de consumo variem. Ainda segundo Zarn, o standard recomendado pelas duas Organizações (LMR) são estritamente baseados nos riscos e não no perigo decorrente de suas consequências para a saúde humana, já que muitos deles são carcinogênicos ou teratogênicos, o que implica o reconhecimento de que standards envolvem valores morais e não apenas econômicos.

A secretaria da OMC, Anneke Hamilton ${ }^{44}$ aponta que o SPS tem dois objetivos principais: reconhecer o direito de proteção da vida e da Saúde humana, dos animais e das plantas e evitar barreiras desnecessárias ao comércio. Assim, se por um lado o SPS tem por objetivo tomar medidas para proteger a vida e saúde humana - animais e plantas - contra a presença de aditivos, contaminantes (pesticidas), toxinas, ou qualquer organismo patogênico em seus alimentos, por outro, busca evita que a diversidade de standards impostos comprometam o livre comercio. No painel apresentado pelo Diretor Nacional de Pesquisa em Resíduos (Austrália), Ian Reichstein, ele mostra dados que parecem comprovar que o

\footnotetext{
${ }^{41}$ BUSCH and BINGEN, 2006, p. 21.

${ }^{42}$ Dias 24 e 25 de outubro de 2016.

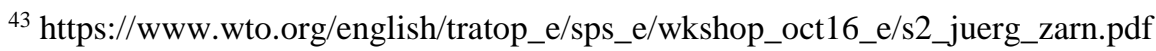

${ }^{44}$ https://www.wto.org/english/tratop_e/sps_e/wkshop_oct16_e/s1_anneke_hamilton.pdf
} 
segundo objetivo ainda prevalece, pois de 2010 a 2016, "many more pesticides and uses are added, than are revoked" 45

\section{CONCLUSÃO}

A proposta deste artigo foi a de enfrentar a problemática da insegurança alimentar no Brasil, no que toca a contaminação dos alimentos por substâncias químicas como herbicidas, pesticidas e outros contaminantes. A temática foi enfrentada a partir da análise, por um lado, dos standards que guiam o sistema alimentar no mundo e por outro, dos indicadores de direitos humanos cuja finalidade é proporcionar um monitoramento efetivo das obrigações do Estado de garantir a segurança alimentar, realizando todas as ações necessárias para a garantia de uma alimentação saudável, sem produtos químicos ou biotecnológicos nocivos à saúde humana.

A insegurança alimentar no Brasil é questão de saúde pública, o que exige um olhar mais atento às questões relacionadas ao monitoramento de direitos humanos, em especial a questão dos indicadores e sua relação com os standards privados de níveis de toxidade.

Neste intento, neste artigo introduziu-se a temática do monitoramento e a problemática dos indicadores de cumprimento das obrigações do Estado na garantia da segurança alimentar, analisando os indicadores de direitos humanos sociais e econômicos da OEA e os propostos nos dois Planos Nacionais de Segurança Alimentar, em especial no que se refere ao monitoramento da utilização de agrotóxicos/pesticidas no Brasil, concluindo-se que indicadores específicos para os agrotóxicos, quando existentes, são totalmente dependentes de standards privados internos e internacionais que estabelecem os níveis de toxidade de acordo com os interesses privados do mercado e que são desenvolvidos cientificamente, utilizando-se de técnicas complexas que muitas vezes comprometem a transparência necessária das informações que contém e que neste sentido estão voltados tanto para evitar riscos à saúde humana, como também para não criar embaraços ao livre comércio das indústrias químicas. Deste modo, sua função como parâmetro para o monitoramento de direitos humanos fica parcialmente prejudicada.

Embora os mercados alternativos estejam ganhando espaço no Brasil, em especial o mercado de produtos frescos e orgânicos, ainda estamos longe de garantir o acesso a todos os brasileiros a uma alimentação não só adequada em termos de quantidade, o que já foi em parte

\footnotetext{
${ }^{45}$ https://www.wto.org/english/tratop_e/sps_e/wkshop_oct16_e/s2_ian_reichstein.pdf
} 
realizado pelos programas de governo ${ }^{46}$, mas em termos de qualidade, o que implica em alimentos livres de qualquer substância, que em qualquer grau possam comprometer a saúde e a vida dos brasileiros. A realização da segurança alimentar depende de mecanismos inovadores, adequados, eficientes e independentes, de monitoramento dos esforços do Brasil na garantia de uma alimentação saudável.

\section{RESULTADOS}

A pesquisa realizada apontou para o problema da dependência dos indicadores de segurança alimentar dos standards privados de níveis de toxidade.

1 - No que refere ao indicador estrutural de recepção do direito do direito à alimentação adequada avaliaria o alcance e abrangência das políticas públicas de qualidade, o que significa levar em conta os standards voltados para o sistema agroalimentar, como o LMR (Limite Máximo de Resíduos em pesticidas).

2 - No que se refere ao indicador "capacidade do Estado" que enseja rever as regras do jogo no aparato do Estado, em especial as habilidades dos agentes que devem estar preparados para enfrentar as exigências técnicas para a compreensão dos standards a serem respeitados. Com relação a este indicador, aponta-se que sua limitação aos padrões emitidos por autoridades do governo, deixando de lado, especificamente, os standards internacionais, que embora não sejam suficientes isoladamente, são necessários para agregar informações.

3 - Em nível do Brasil, constatou-se que todas as análises feitas pelo implicam diretamente em standards de toxicidade. No mesmo sentido o indicador proposto pelo PLANSAN, para substâncias químicas que se refere ao percentual de Contaminação de alimentos por agrotóxicos (amostras irregulares).

4 - No PLANAN 2016-2019, observou-se a meta de reavaliação de 11 ingredientes ativos de produtos agrotóxicos já registrados, considerando novos indícios de risco à saúde humana e a revisão da a norma que determina os procedimentos para reavaliação toxicológica de ingredientes ativos de agrotóxicos com novos indícios de riscos à saúde humana (RDC Anvisa n ${ }^{\circ} 48 / 2008$ ), que implicam na dependência dos standards de nível de toxidade

\section{REFERÊNCIAS}


Busch L; Bingen J. The world of standards. In: Korthals, $M$ and Thompson, PB. Agricultural Standards. The shape of the global food and fiber system. Volume 6. Springer. 2006.

Câmara Interministerial de Segurança Alimentar e Nutricional (BR). Plano Nacional de Segurança Alimentar e Nutricional 2012-2015. Brasília, DF. 2011.

(BR). Indicadores e Principais Resultados do Plano Nacional de Segurança Alimentar e Nutricional. Brasília. DF. 2015.

(BR). Plano Nacional de Segurança Alimentar e Nutricional 2016-2019.

2016

Faria N., Fasse ACG. e Fachini LA. Intoxicação por agrotóxicos no Brasil: os sistemas oficiais de informações e desafios para a realização de estudos epidemiológicos. Ciência e saúde coletiva, v.12 n.1 Rio de Janeiro jan./mar.http://dx.doi.org/10.1590/S141381232007000100008.2007 .

Fligstein N. The end of (shareholder value) ideology? Political Power and Social Theory 17, pgs 223-228. January. 2005.

Green M. What we talk about indicators: current approaches to human Rights Measurement. Human Rights Quaterly, vol. 23, number 4, pp. 1062-1097. The Johns Hopkins University Press. 2001.

Ilcan S and Phillips L. Circulations of insecurity: globalizing food Standards in Historical Perspective1 in Korthals $M$ and Thompson P.B. Agricultural Standards. The shape of the global food and fiber system, Volume 6. Springer. 2006.

Ibama (BR). Boletim de Comercialização de Agrotóxicos e Afins: Histórico de vendas 2000 a 2009. Brasília. DF. 2012

Ipes Food. International panel of experts on sustainable food systems. From uniformity to diversity: A paradigm shift from industrial agriculture to diversified agroecological systems. Report 2. 2016.

American States Organization. A Guidelines for preparation of progress indicators in the area of economic, social and cultural rights. OEA/Ser. L/V/II.132. doc. 14.19 july, 2008. Available electronically http://www.cidh.oas.org/pdf\%20files/Guideline\%20october\%202007\%20eng.pdf. 2008.

Indicadores de progreso para medición de derechos contemplados en el Protocolo de San Salvador. OEA/Ser.L/XXV.2.1 GT/PSS/doc.2/11 rev.2 16 diciembre 2011 Original: $\quad$ Español. Available electronically via http://www.sedi.oas.org/ddse/documentos/Protocolo\%20San\%20Salvador/11.\%20ESP_\%20I ndicadoresProgreso-rev2.pdf. 2011.

Progress indicators for measuring rigts contemplated in the Additional Protocol to the American Convention on Human Rights in the Area of Economic, Social, and Cultural Rights,"Protocol of San Salvador / [Prepared by the Working Group to 
examine the periodic reports of the States Parties to the Protocol of San Salvador]. p.; cm. (OAS. Official records; OEA/Ser.D) ISBN 978-0-8270-6421-8. 2015. Available electronically via http://socialprotectionet.org/sites/default/files/escrs_indicators.pdf. 2015.

United Nations. Organization for Food and Agriculture. FAO specifications and evaluations for agricultural pesticides. GLYPHOSATE (N-(phosphonomethyl)glycine). 2016.

Organization for Food and Agriculture and World Health Organization. Report of the Joint Meeting of the FAO Panel of Experts on Pesticide Residues in Food and the Environment and the WHO Core Assessment Group on Pesticide Residues Rome, Italy, 21-30 September. 2010.

Organisation for Economic Co-operation and Development. Environmental Outlook for the Chemicals Industries. Available via http://www.oecd.org/env/ehs/2375538.pdf. 2001.

$\begin{array}{cccc} & \text { Environmental outlook to 2030. ISBN } & 978-92-64-04048-9 . & \text { Available } \\ \text { electronically } & \text { via } & \text { http://www.oecd.org/environment/indicators-modelling- }\end{array}$ outlooks/40200582.pdf. 2008.

/FAO. Perspectivas agrícolas 2015-2024. 2015.

Randolph S. and Hertel S. A right to food: a global overview. Working Papers 19. Economic Rights Working Paper Series. Human Rights Institute. University of Connecticut. http://www.econ.uconn.edu/working/19.pdf. 2012.

and Fukuda-Parr S. \& Lawson-Remer T. Economic and Social Rights Fulfillment Index: Country Scores and Rankings. Working papers 27, University of Connecticut, Department of Economics. USA. 2009.

and Guyer P. Tracking the Historical Evolution of States' compliance with their Economic and Social Rights Obligations of Result: Insights from the Historical SERF Index. Working Papers 18. Economic Rights Working Paper Series. Human Rights Institute. University of Connecticut. http://www.econ.uconn.edu/working/18.pdf. 2012.

Siqueira S. e Kruse M. Agrotóxicos e saúde humana: contribuição dos profissionais do campo da saúde. Ver. Esc. Enfermagem USP. Encontrado em http://www.scielo.br/pdf/reeusp/v42n3/v42n3a23.pdf. 2008.

Téran AS. Progressividad y creación de indicadores para medir el cumprimento de los DESC en México. Indicadores de desarollo e indicadores de derechos humanos. Instituto de Investigationes Juridicas de la UNAM. Ponencia presentada, em agosto de 2005, durante el Seminário Internacional sobre Derechos Ecoômicos, Sociales y Culturales (DESC), realizado em instalaciones de la SER, em Tlateloco. F.F. 2005.

Unites Nations. Guiding Principles on Business and Human Rights: Implementing the United Nations "Protect, Respect and Remedy. Report of the Special Representative of the Secretary-General on the issue of human rights and transnational corporations and other business enterprises, John Ruggie. 2011. 
Human Rights Office of the Hight Comissioner. Indicators. A Guide

to Measurement and Implementation. Available electronically
http://www.ohchr.org/Documents/Issues/HRIndicators/AGuideMeasurementImplementationC ompleteGuide_en.pdf.pdf. 2012.

Indicators and a Monitoring Framework for the Sustainable Development Goals Launching a data revolution for the SDGs. A report by the Leadership Council of the Sustainable Development Solutions Network Revised working draft (Version 6). February 18, Available electronically via http://unsdsn.org/wpcontent/uploads/2015/05/150612-FINAL-SDSN-Indicator-Report1.pdf. 2014. 